\title{
Vector Field Path Following for Miniature Air Vehicles
}

Timothy McLain

Mechanical Engineering Department, Brigham Young University, mclain@byu.edu

Derek R. Nelson

Unmanned Systems Division of Northrop Grumman

D. Blake Barber

Mechanical Engineering Department, Brigham Young University

Randall W. Beard

Department of Electrical Engineering, Brigham Young University

Follow this and additional works at: https://scholarsarchive.byu.edu/facpub

Part of the Mechanical Engineering Commons

\section{Original Publication Citation}

Nelson, D., Barber, B., McLain, T., and Beard, R. Vector Field Path Following for Miniature Air Vehicles, IEEE Transactions on Robotics, vol. 23, no. 3, pp. 519-529, June 2007.

\section{BYU ScholarsArchive Citation}

McLain, Timothy; Nelson, Derek R.; Barber, D. Blake; and Beard, Randall W., "Vector Field Path Following for Miniature Air Vehicles" (2007). Faculty Publications. 1902.

https://scholarsarchive.byu.edu/facpub/1902 


\title{
Vector Field Path Following for Miniature Air Vehicles
}

\author{
Derek R. Nelson, D. Blake Barber, Timothy W. McLain, Senior Member, IEEE and \\ Randal W. Beard. Senior Member, IEEE
}

\begin{abstract}
In this paper, a method for accurate path following for miniature air vehicles is developed. The method is based on the notion of vector fields, which are used to generate desired course inputs to inner-loop attitude control laws. Vector field path following control laws are developed for straight-line paths and circular arcs and orbits. Lyapunov stability arguments are used to demonstrate asymptotic decay of path following errors in the presence of constant wind disturbances. Experimental flight tests have demonstrated mean path following errors on less than one wingspan for straight-line and orbit paths, and less than three wingspans for paths with frequent changes in direction.
\end{abstract}

\section{INTRODUCTION}

Unmanned aerial vehicles (UAVs), large and small, have demonstrated their usefulness in military applications. Furthermore, there are numerous potential uses for UAVs in civil and commercial applications and the prospects for broad impact are strong. To extend the usefulness of UAVs beyond their current applications, the capability to plan paths and to follow them accurately is of great importance. Unlike piloted vehicles, which rely on the pilot to navigate over demanding terrain or to avoid obstructions, UAVs rely on automation to provide this functionality. As applications such as urban surveillance and rural search and rescue require UAVs to fly down city streets surrounded by buildings or near the surface of abruptly changing mountainous terrain, the ability to follow pre-planned paths with precision is essential. For missions involving cooperation among a team of UAVs, precise path tracking is often crucial to achieving the cooperation objective.

For miniature aerial vehicles, ${ }^{1}$ such as those of primary interest in this work, wind disturbances, dynamic characteristics, and the quality of sensing and control all limit the achievable tracking precision. For MAVs wind speeds are commonly 20 to 60 percent of the desired airspeed. Effective path tracking strategies must overcome the effect of this ever present disturbance. For most fixed-wing MAVs, the minimum turn radius is in the range of 10 to $50 \mathrm{~m}$. This places a

Manuscript submitted December, 2005.

D. Nelson is with the Unmanned Systems Division of Northrop Grumman Corp. B. Barber is a graduate research assistant in the Mechanical Engineering Department at Brigham Young University. T. W. McLain is an associate professor in the Mechanical Engineering Department at Brigham Young University. R. W. Beard is an associate professor in the Electrical and Computer Engineering Department at Brigham Young University.

This work was funded by AFRL/MNK grant F08630-03-1-0017 and by AFOSR grants FA9550-04-1-0209 and FA9550-04-C-0032.

T. W. McLain is the corresponding author: mclain@byu.edu

${ }^{1}$ We consider miniature aerial vehicles to be those with wingspans in the $0.3 \mathrm{~m}$ to $2 \mathrm{~m}$ range and micro aerial vehicles to be those with wingspans under $0.3 \mathrm{~m}$. Here the abbreviation MAV denotes miniature aerial vehicle. fundamental limit on the spatial frequency of paths that can be tracked. Thus, it is important that the path tracking algorithms utilize the full capability of the MAV. Finally, high-resolution state sensors with high-frequency updates are not typically available for MAVs. Successful tracking approaches must exploit fully those sensors that are readily available.

Several approaches have been proposed for UAV trajectory tracking. An approach for tight tracking of curved trajectories is presented in [1]. For straight-line paths, the approach approximates PD control. For curved paths, an additional anticipatory control element that improves the tracking capability is implemented. The approach accommodates the addition of an adaptive element to account for disturbances such as wind. This approach is validated with flight experiments.

In [2], Kaminer et al. describe an integrated approach for developing guidance and control algorithms for autonomous vehicle trajectory tracking. Their approach builds upon the theory of gain scheduling and produces controllers for tracking trajectories that are defined in an inertial reference frame. The approach is illustrated through simulations of a small UAV.

Implicit in the notion of trajectory tracking is that the vehicle is commanded to be in a particular location at a particular time and that this location typically varies in time, thus causing the vehicle to move in the desired fashion. With fixed-wing MAVs, the desired position is constantly moving (at the desired ground speed). The approach of tracking a moving point can result in significant problems for MAVs if disturbances, such as those due to wind, are not accounted for properly. If the MAV is flying into a strong wind (relative to its commanded ground speed), the progression of the trajectory point must be slowed accordingly. Similarly, if the MAV is flying down wind, the speed of the tracking point must be increased to keep the MAV from overrunning the desired position. Given that wind disturbances vary and are often not easily predicted, trajectory tracking can be very challenging in anything other than calm conditions.

Rather than pursuing the trajectory tracking approach, this paper explores path following where the objective is to be on the path rather than at a certain point at a particular time. With path following, the time dependence of the problem is removed. In [3], [4], performance limits for referencetracking and path-following controllers are investigated and the difference between them is highlighted. It is shown that there is not a fundamental performance limitation for path following for systems with unstable zero dynamics as there is for reference tracking.

Building on the work presented in [5] on maneuver mod- 
ified trajectory tracking, Encarnação and Pascoal develop an approach that combines the features of trajectory tracking and path following for marine vehicles [6]. Similar to this work is that of Skjetne, et al. [7] which develops an output maneuvering method composed of two tasks: forcing the output to converge to the desired path and then satisfying a desired speed assignment along the path. The method is demonstrated using a marine vessel simulation. Ref. [8] presents a path following method for UAVs that provides a constant line of sight between the UAV and an observation target.

The work presented in this paper builds on the concept of path following through the construction of vector fields surrounding the path to be followed. The vectors of the fields provide course commands to guide the MAV toward the desired path. As with other path following methods, the objective is not to track a moving point, but to get onto the path while flying at a prescribed airspeed. A unique contribution of this paper is the utilization of course measurements in the path following control, which in combination with the vector field strategy, guarantees that tracking errors asymptotically approach zero even in the presence of constant wind disturbances. Implementation of the approach is feasible on small MAVs and experimental results validate the potential value of the approach for MAVs flying in windy conditions.

\section{Problem Description}

The objective of this paper is to develop a method for accurate path following for MAVs in the presence of wind. The method calculates a vector field around the path to be tracked. The vectors in the field are directed toward the path to be followed and represent the desired direction of flight. The vectors in the field serve as course commands to the MAV. The method is currently applicable to paths composed of straight lines and arcs. This restriction is insignificant for most practical applications. Figure 1 shows examples of vector fields for linear and circular paths.
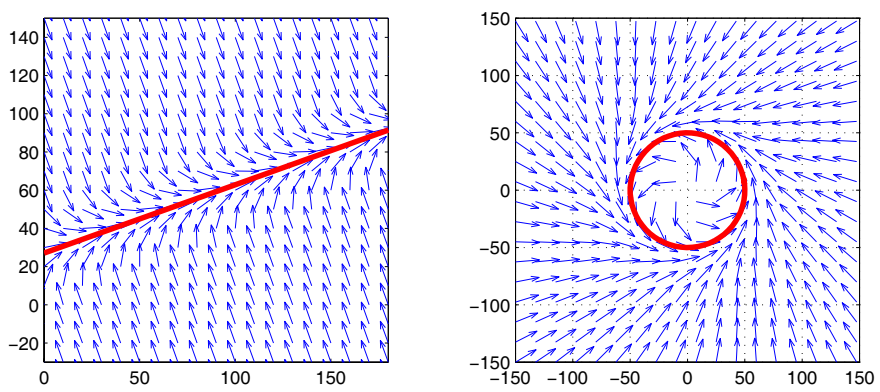

Fig. 1. This figure illustrates the vector field idea for straight line and circular path following. The the desired course of the MAV is specified the by direction of the vector field.

The notion of vector fields is similar to that of potential fields, which have been widely used as a tool for path planning in the robotics community (see e.g., [9]). It has also been suggested in [10] that potential fields can be used in UAV navigation for obstacle and collision avoidance applications. The method of [10] provides a way for groups of UAVs to use the gradient of a potential field to navigate through heavily populated areas safely while still aggressively approaching their targets. Vector fields are different from potential fields in that they do not necessarily represent the gradient of a potential. Rather, the vector field simply indicates a desired direction of travel. In [11], the concept of vector fields is used to direct autonomous aircraft in the stand-off tracking of a moving target. Precision tracking of a predefined path is not considered. Instead, the vector field is utilized to direct a team of two aircraft into an orbit around a moving target.

This paper considers the navigation of a fixed-wing MAV with the assumption that altitude and airspeed $\left(V_{a}\right)$ are held constant (or nearly so) by the control of the longitudinal dynamics. The following is a simple model of the navigational dynamics that will be used to study the path following behavior of the proposed approach:

$$
\begin{aligned}
& \dot{x}=V_{a} \cos \psi+W_{x} \\
& \dot{y}=V_{a} \sin \psi+W_{y}
\end{aligned}
$$

where $\left(W_{x}, W_{y}\right)$ represent the $x$ and $y$ components of the wind velocity. Heading $(\psi)$ will be controlled by the vector field path following approaches presented in this paper. An alternative representation of these equations can be developed by noting the relationship between groundspeed $\left(V_{g}\right)$, airspeed $\left(V_{a}\right)$, and wind speed $(W)$ as depicted in Figure 2:

$$
\begin{aligned}
& \dot{x}=V_{a x}+W_{x}=V_{g x} \\
& \dot{y}=V_{a y}+W_{y}=V_{g y} .
\end{aligned}
$$

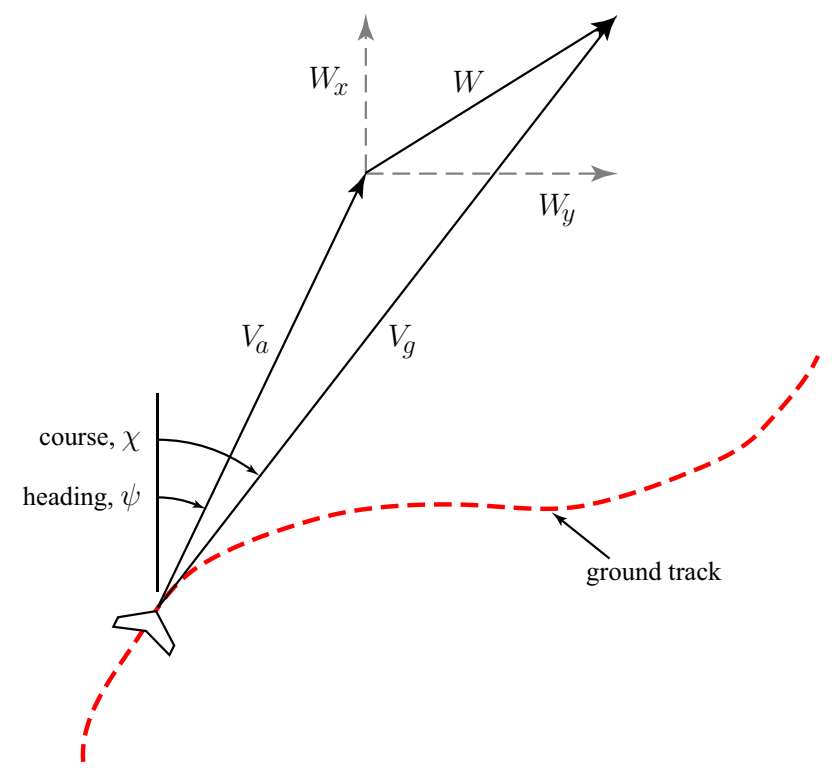

Fig. 2. This figure shows the relationship between the airspeed $V_{a}$, the windspeed $W$, and ground speed $V_{g}$, as well as the relationship between heading $\psi$ and course $\chi$.

Drawing on (3) and (4) and the definition of course $(\chi)$ shown in Figure 2, (1) and (2) can be expressed as

$$
\begin{aligned}
& \dot{x}=V_{g} \cos \chi \\
& \dot{y}=V_{g} \sin \chi
\end{aligned}
$$


The key distinction is that the equations of motion are expressed in terms of groundspeed and course and are independent of the wind velocity. We will show that by using groundreferenced measurements (i.e., course and groundspeed instead of heading and airspeed) in conjunction with the vector field approach to control the path of the vehicle, wind-disturbance rejection is improved significantly, which is vitally important for small, low-speed MAVs. We will assume that the MAV is equipped with an autopilot that implements a course-hold loop and that the resulting dynamics are represented by the first-order system

$$
\dot{\chi}=\alpha\left(\chi^{c}-\chi\right),
$$

where $\chi^{c}$ is the commanded course, and $\alpha$ is a known positive constant that characterizes the speed of response of coursehold autopilot loop.

In the development and analysis of the vector field approach that follows, two primitive path types are considered: straight lines and circular orbits. Circular arcs are treated similarly to complete orbits. The approach is easily extended to paths composed of multiple segments of arcs, orbits, and straight lines.

\section{TECHNICAL APPROACH}

\section{A. Straight Path Following}

Consider the straight-line path shown in Figure 3. To follow this path, a desired-course vector field is constructed. Let $y$ be the lateral distance of the MAV from the path, and let $\chi$ be the difference between the direction of the path and the course of the MAV. Our objective is to construct the vector field so that when $y$ is large the MAV is directed to approach the path with course angle $\chi^{\infty}$, and that as $y$ approaches zero, the course $\chi$ also approaches zero. Toward that end, define the desired course of the MAV as

$$
\chi^{d}(y)=-\chi^{\infty} \frac{2}{\pi} \tan ^{-1}(k y),
$$

where $k$ is a positive constant that influences the rate of the transition from $\chi^{\infty}$ to zero. Figure 4 shows how the choice of $k$ affects the rate of transition. Large values of $k$ result in short, abrupt transitions, while small values of $k$ cause long, smooth transitions in the desired course.

If $\chi^{\infty}$ is restricted to be in the range $\chi^{\infty} \in\left(0, \frac{\pi}{2}\right]$ then clearly

$$
-\frac{\pi}{2}<\chi^{\infty} \frac{2}{\pi} \tan ^{-1}(k y)<\frac{\pi}{2}
$$

for all values of $y$. Therefore since $\tan ^{-1}(\cdot)$ is an odd function and $\sin (\cdot)$ is odd over $\left(-\frac{\pi}{2}, \frac{\pi}{2}\right)$ we can use the Lyapunov function $W_{1}(y)=\frac{1}{2} y^{2}$ to argue that if $\chi=\chi^{d}(y)$, then $y \rightarrow 0$ asymptotically. Evaluating the Lie derivative of $W_{1}$ under the assumption that $\chi=\chi^{d}(y)$ gives

$$
\begin{aligned}
\dot{W}_{1} & =V_{g} y \sin \left(\chi^{d}(y)\right) \\
& =-V_{g} y \sin \left(\chi^{\infty} \frac{2}{\pi} \tan ^{-1}(k y)\right),
\end{aligned}
$$

which is less than zero for $y \neq 0$.

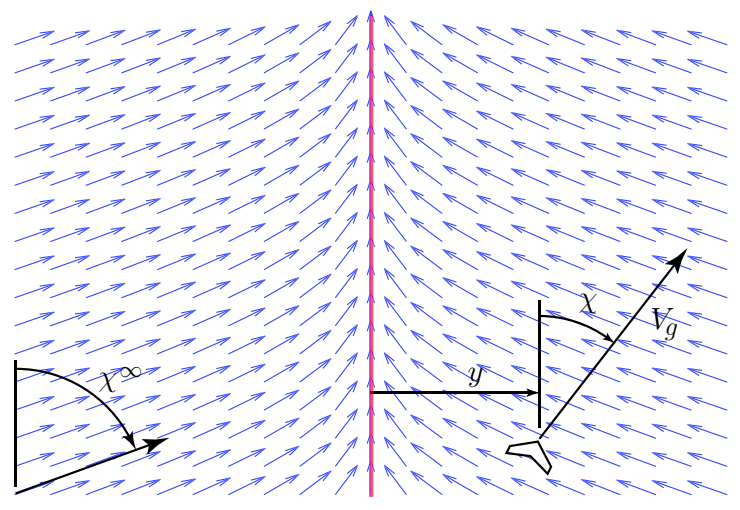

Fig. 3. Vector field for straight-line path following. Far away from the waypoint path, the vector field is directed with an angle $\chi^{\infty}$ from the perpendicular to the path.

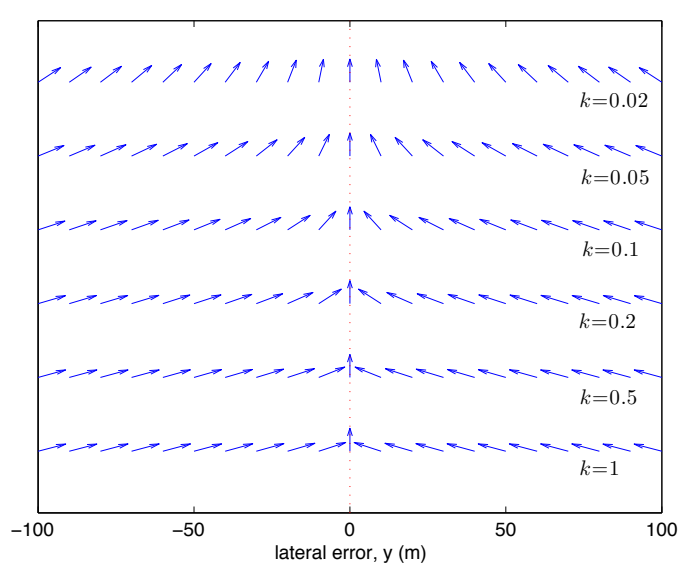

Fig. 4. Vector fields for various values of $k$. Large values of $k$ yield abrupt transitions from $\chi^{\infty}$ to zero, while small values of $k$ give smooth transitions.

In this paper we use a sliding mode approach to render the set

$$
S=\left\{(y, \chi): \chi=\chi^{d}(y)\right\}
$$

positively invariant and to ensure that the system trajectory reaches $S$ in finite time. Let $\tilde{\chi} \triangleq \chi-\chi^{d}(y)$ and differentiate to obtain

$$
\begin{aligned}
\dot{\tilde{\chi}} & =\dot{\chi}-\dot{\chi}^{d}(y) \\
& =\alpha\left(\chi^{c}-\chi\right)+\chi^{\infty} \frac{2}{\pi} \frac{k}{1+(k y)^{2}} V_{g} \sin \chi .
\end{aligned}
$$

Let $W_{2}=\frac{1}{2} \tilde{\chi}^{2}$ and take the derivative to obtain

$$
\begin{aligned}
\dot{W}_{2} & =\tilde{\chi} \dot{\tilde{\chi}} \\
& =\tilde{\chi}\left(\dot{\chi}-\dot{\chi}^{d}(y)\right) \\
& =\tilde{\chi}\left(\alpha\left(\chi^{c}-\chi\right)+\chi^{\infty} \frac{2}{\pi} \frac{k}{1+(k y)^{2}} V_{g} \sin \chi\right) .
\end{aligned}
$$

If we choose the control signal as

$$
\chi^{c}=\chi-\frac{1}{\alpha} \chi^{\infty} \frac{2}{\pi} \frac{k}{1+(k y)^{2}} V_{g} \sin \chi-\frac{\kappa}{\alpha} \operatorname{sign}(\tilde{\chi}),
$$


where

$$
\operatorname{sign}(x)= \begin{cases}1 & \text { if } x>0 \\ 0 & \text { if } x=0 \\ -1 & \text { if } x<0\end{cases}
$$

and $\kappa>0$, then

$$
\dot{W}_{2} \leq-\kappa|\tilde{\chi}|
$$

from which we conclude that $\tilde{\chi} \rightarrow 0$ in finite time [12].

It is well known that the sign function leads to chattering in the control [12]. To mitigate the adverse effects of chattering, the control signal (10) is replaced with

$$
\chi^{c}=\chi-\frac{1}{\alpha} \chi^{\infty} \frac{2}{\pi} \frac{k}{1+(k y)^{2}} V_{g} \sin \chi-\frac{\kappa}{\alpha} \operatorname{sat}\left(\frac{\tilde{\chi}}{\epsilon}\right),
$$

where

$$
\operatorname{sat}(x)=\left\{\begin{array}{ll}
x & \text { if }|x| \leq 1 \\
\operatorname{sign}(x) & \text { otherwise }
\end{array},\right.
$$

and $\epsilon>0$ defines the width of the boundary region around the sliding surface.

The shape of the sliding surface and system trajectory onto the sliding surface are influenced by the control parameters $k$, $\chi^{\infty}, \kappa$, and $\epsilon$. Figure 5 shows state trajectories onto the sliding surface $\chi^{d}(y)$ for various initial conditions generated from (6), (7), (8), and (12). The parameter $k$ controls the slope of the sliding surface near the origin, while $\chi^{\infty}$ scales the magnitude of $\chi^{d}(y)$ for large values of $y$. The gain parameter $\kappa$ controls the shape of the trajectories onto the sliding surface. Large values of $\kappa$ drive $\tilde{\chi}$ to zero quickly. The parameter $\epsilon$ is the width of the transition region around the sliding surface that is used to reduce chatter in the control.

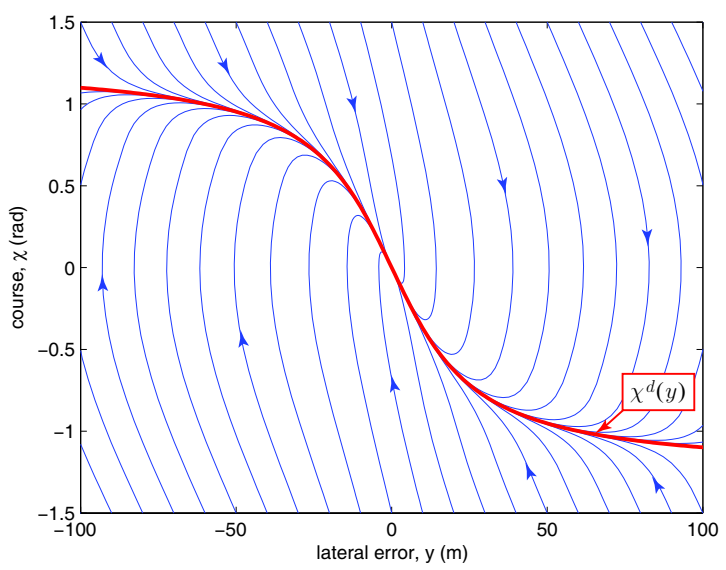

Fig. 5. State trajectories onto the sliding surface $\chi^{d}(y)$ for various initial conditions.

Theorem III.1 The system of equations given by (6) and (9), where $\chi^{c}$ is given by (12) and $\chi^{d}(y)$ is given by (8) is globally exponentially stable if $0<\chi^{\infty} \leq \frac{\pi}{2}$ and

$$
\left(\frac{2 \chi^{\infty} k}{\pi \epsilon \mu}\right) \min \left\{\left(\frac{\rho \kappa}{V_{g}}\right),\left(\frac{\bar{y}}{2}\right)\right\}>1,
$$

where

$\mu \geq \max \left\{\frac{2\left(1+(k \bar{y})^{2}\right)}{\cos \left(\frac{2 \chi^{\infty}}{\pi} \tan ^{-1}(k \bar{y})\right)}, \frac{4 \chi^{\infty} k \bar{y}}{\pi \sin \left(\frac{2 \chi^{\infty}}{\pi} \tan ^{-1}(k \bar{y})\right)}\right\}$

and $\bar{y}$ is arbitrary.

The stability condition (13) makes sense physically. If $V_{g}$ is large, than the turning radius of the MAV increases making it more difficult to remain within the boundary layer. Similarly small $\chi^{\infty}$ or small $k$ imply slower convergence toward the path as indicated by Figures 3 and 4 . Small $\epsilon$ makes the feedback control high gain which enhances tracking. It is interesting to note that (13) is independent of $\alpha$. However, from (12) it is clear that to avoid unrealistic control effort, $\kappa$ is required to be proportional to $\alpha$. Therefore, (13) is easier to satisfy if $\alpha$ is large, implying that the on-board autopilot can quickly track course changes.

Proof:

If $|\tilde{\chi}| \geq \epsilon$, then $\chi^{c}$ is equivalent to (10) which results in (11) implying that the set

$$
S_{\epsilon} \triangleq\{|\tilde{\chi}| \leq \epsilon\}
$$

is positively invariant and that $\tilde{\chi}$ converges to $S$ in finite time.

It remains to show that inside $S_{\epsilon}$ the system trajectories converge to the origin $(y, \tilde{\chi})=(0,0)$. Toward that end define the Lypunov function candidate

$$
W=\frac{1}{2} y^{2}+\frac{1}{2} \rho \tilde{\chi}^{2}
$$

where $\rho$ is a positive scale factor used to weight the terms so that they are similar in magnitude. Differentiating we obtain

$$
\begin{aligned}
\dot{W}=y \dot{y} & +\rho \tilde{\chi} \dot{\tilde{\chi}} \\
=y & V_{g} \sin \left(\chi^{d}(y)+\tilde{\chi}\right) \\
& \quad+\rho \tilde{\chi} \alpha\left(\chi^{c}-\chi+\frac{1}{\alpha} \frac{2 \chi^{\infty}}{\pi} \frac{k}{1+(k y)^{2}} V_{g} \sin \chi\right) .
\end{aligned}
$$

Inside the boundary region we have

$$
\chi^{c}=\chi-\frac{1}{\alpha} \chi^{\infty} \frac{2}{\pi} \frac{k}{1+(k y)^{2}} V_{g} \sin \chi-\frac{\kappa}{\alpha} \frac{\tilde{\chi}}{\epsilon} .
$$

Therefore

$$
\begin{aligned}
\dot{W}= & V_{g} y \sin \left(\chi^{d}(y)+\tilde{\chi}\right)-\frac{\rho \kappa}{\epsilon} \tilde{\chi}^{2} \\
=-\frac{\rho \kappa}{\epsilon} & \tilde{\chi}^{2}+V_{g} y \sin \left(\chi^{d}(y)\right) \\
& \quad+V_{g} y\left(\sin \left(\chi^{d}(y)+\tilde{\chi}\right)-\sin \left(\chi^{d}(y)\right)\right) \\
\leq-\frac{\rho \kappa}{\epsilon} & \tilde{\chi}^{2}+V_{g} y \sin \left(\chi^{d}(y)\right) \\
& \quad+V_{g}|y|\left|\sin \left(\chi^{d}(y)+\tilde{\chi}\right)-\sin \left(\chi^{d}(y)\right)\right| .
\end{aligned}
$$

Noting that

$$
\begin{aligned}
\mid \sin & \left(\chi^{d}(y)+\tilde{\chi}\right)-\sin \left(\chi^{d}(y)\right) \mid \\
& =\left|\sin \chi^{d}(y) \cos \tilde{\chi}+\cos \chi^{d}(y) \sin \tilde{\chi}-\sin \chi^{d}(y)\right| \\
& =\left|\sin \chi^{d}(y)(\cos \tilde{\chi}-1)+\cos \chi^{d}(y) \sin \tilde{\chi}\right| \\
& \leq|\cos \tilde{\chi}-1|+|\sin \tilde{\chi}| \\
& \leq 2|\tilde{\chi}|,
\end{aligned}
$$


we get

$$
\begin{aligned}
\dot{W} & \leq-\frac{\rho \kappa}{\epsilon} \tilde{\chi}^{2}+2 V_{g}|y||\tilde{\chi}|+V_{g} y \sin \left(\chi^{d}(y)\right) \\
& =-\frac{\rho \kappa}{\epsilon} \tilde{\chi}^{2}+2 V_{g}|y||\tilde{\chi}|-V_{g} y \sin \left(\frac{2 \chi^{\infty}}{\pi} \tan ^{-1}(k y)\right) .
\end{aligned}
$$

Let

$$
\phi(y)=y \sin \left(\frac{2 \chi^{\infty}}{\pi} \tan ^{-1}(k y)\right),
$$

and note that $\phi(y) \approx \frac{2 \chi^{\infty} k}{\pi} y^{2}$ for small values of $k y$ and $\phi(y) \approx\left(\sin \chi^{\infty}\right) y$ for large $k y$. Consider the function

$$
\varphi(y)= \begin{cases}\frac{2 \chi^{\infty} k}{\mu \pi} y^{2} & \text { if }|y| \leq \bar{y} \\ \frac{2 \chi^{\infty} k \bar{y}}{\mu \pi}(2|y|-\bar{y}) & \text { if }|y|>\bar{y}\end{cases}
$$

where $\bar{y}$ is arbitrary. Our objective is to find $\mu$ that ensures that $0<\varphi(y) \leq \phi(y)$.

Toward that end, note that both $\phi$ and $\varphi$ are symmetric functions in $y$. Therefore, without loss of generality we will restrict our attention to $y \geq 0$. To show that $\varphi(y) \leq \phi(y)$ we will use the fact that if two functions $f$ and $g$ satisfy $f(0)=g(0)$ and $f^{\prime}(y) \leq g^{\prime}(y)$ for $y \geq 0$, then $f(y) \leq g(y)$ for $y \geq 0$. Toward that end, note that when $0 \leq y \leq \bar{y}$

$$
\begin{aligned}
\phi^{\prime}(y)= & \sin \left(\frac{2 \chi^{\infty}}{\pi} \tan ^{-1}(k y)\right) \\
& \quad+\frac{2 \chi^{\infty} k}{\pi} y\left[\frac{\cos \left(\frac{2 \chi^{\infty}}{\pi} \tan ^{-1}(k y)\right)}{1+(k y)^{2}}\right] \\
\geq & \frac{4 \chi^{\infty} k}{\pi} y\left[\frac{1}{2} \frac{\cos \left(\frac{2 \chi^{\infty}}{\pi} \tan ^{-1}(k y)\right)}{1+(k y)^{2}}\right] \\
\geq & \frac{4 \chi^{\infty} k}{\pi} y\left[\frac{1}{2} \frac{\cos \left(\frac{2 \chi^{\infty}}{\pi} \tan ^{-1}(k \bar{y})\right)}{1+(k \bar{y})^{2}}\right] \\
\geq & \frac{4 \chi^{\infty} k}{\mu \pi} y \\
= & \varphi^{\prime}(y)
\end{aligned}
$$

if

$$
\mu \geq \frac{2\left(1+(k \bar{y})^{2}\right)}{\cos \left(\frac{2 \chi^{\infty}}{\pi} \tan ^{-1}(k \bar{y})\right)} .
$$

Also note that when $y>\bar{y}$, we have

$$
\begin{aligned}
\phi(y) & =y \sin \left(\frac{2 \chi^{\infty}}{\pi} \tan ^{-1}(k y)\right) \\
& \geq y \sin \left(\frac{2 \chi^{\infty}}{\pi} \tan ^{-1}(k \bar{y})\right)
\end{aligned}
$$

which implies that $\phi(y) \geq \varphi(y)$ if

$$
\frac{4 \chi^{\infty} k \bar{y}}{\mu \pi} \leq \sin \left(\frac{2 \chi^{\infty}}{\pi} \tan ^{-1}(k \bar{y})\right)
$$

which is equivalent to

$$
\mu \geq \frac{4 \chi^{\infty} k \bar{y}}{\pi \sin \left(\frac{2 \chi^{\infty}}{\pi} \tan ^{-1}(k \bar{y})\right)}
$$

Therefore, if

$\mu \geq \max \left\{\frac{2\left(1+(k \bar{y})^{2}\right)}{\cos \left(\frac{2 \chi^{\infty}}{\pi} \tan ^{-1}(k \bar{y})\right)}, \frac{4 \chi^{\infty} k \bar{y}}{\pi \sin \left(\frac{2 \chi^{\infty}}{\pi} \tan ^{-1}(k \bar{y})\right)}\right\}$

then $\varphi(y) \leq \phi(y)$ which implies that

$$
\dot{W} \leq-\frac{\rho \kappa}{\epsilon} \tilde{\chi}^{2}+2 V_{g}|y||\tilde{\chi}|-V_{g} \varphi(y)
$$

Therefore, for $|y| \leq \bar{y}$ we get

$$
\begin{aligned}
& \dot{W} \leq-\frac{\rho \kappa}{\epsilon} \tilde{\chi}^{2}+2 V_{g}|y||\tilde{\chi}|-\frac{2 V_{g} \chi^{\infty} k}{\mu \pi} y^{2}
\end{aligned}
$$

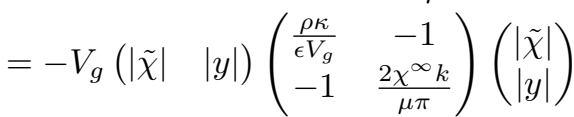

which is negative definite if

$$
\frac{\rho \kappa}{\epsilon V_{g}}\left(\frac{2 \chi^{\infty} k}{\mu \pi}\right)>1
$$

Exponential stability comes from the fact that $W$ and the right hand side of (14) are quadratic [12].

For $|y|>\bar{y}$, we get

$$
\begin{aligned}
\dot{W} & \leq-\frac{\rho \kappa}{\epsilon} \tilde{\chi}^{2}+2 V_{g}|y||\tilde{\chi}|-\frac{2 V_{g} \chi^{\infty} k \bar{y}}{\mu \pi}(2|y|-\bar{y}) \\
& \leq 2 V_{g}|y| \epsilon-\frac{2 V_{g} \chi^{\infty} k \bar{y}}{\mu \pi}|y| \\
& =2 V_{g}|y|\left(\epsilon-\frac{\chi^{\infty} k \bar{y}}{\mu \pi}|y|\right)
\end{aligned}
$$

which is less than zero if

$$
\frac{\chi^{\infty} k \bar{y}}{\mu \epsilon \pi}>1,
$$

thus implying asymptotic stability for $|y|>\bar{y}$.

\section{B. Orbit Following}

The algorithm for circular orbits creates vector fields in a manner similar to the straight-line algorithm. Consider the desired orbit path shown in Figure 6. In this discussion, a counter-clockwise orbit will be considered. The development for clockwise orbits is similar with the exception of several sign changes. The desired orbit is assumed to have a known center and radius $r$. When the distance between the MAV and the center of the orbit is large, it is desirable for the MAV to fly toward the orbit center. If we define $d$ as the radial distance of the MAV from the center of the orbit, then when $d$ is significantly larger than $r$ the desired course is

$$
\chi^{d} \approx \gamma-\pi
$$

where $\gamma$ is defined as the angular position of the MAV with respect to the orbit center as shown in Figure 6.

When $d=r$ the desired course is $\chi^{d}=\gamma-\frac{\pi}{2}$. Therefore let the desired course be given by

$$
\chi^{d}(d)=\gamma-\frac{\pi}{2}-\tan ^{-1}(k(d-r)),
$$

where $k>0$ is a constant that specifies the rate of transition from $\gamma-\pi$ to $\gamma-\frac{\pi}{2}$. This expression for $\chi^{d}$ is valid for all values of $d \geq 0$. 
For orbit following, it is convenient to change the navigational dynamics to polar coordinates in terms of $d$ and $\gamma$ where the center of the orbit is the origin. From Figure 6, $x=c_{x}+d \cos \gamma$ and $y=c_{y}+d \sin \gamma$, where $\left(c_{x}, c_{y}\right)$ is the center of the orbit. Taking the derivative and substituting into Equations (5) and (6) gives

$$
\begin{aligned}
& \dot{d}=V_{g} \cos (\chi-\gamma) \\
& \dot{\gamma}=\frac{V_{g}}{d} \sin (\chi-\gamma)
\end{aligned}
$$

where the $V_{g}$ and $\chi$ are the ground track speed and relative

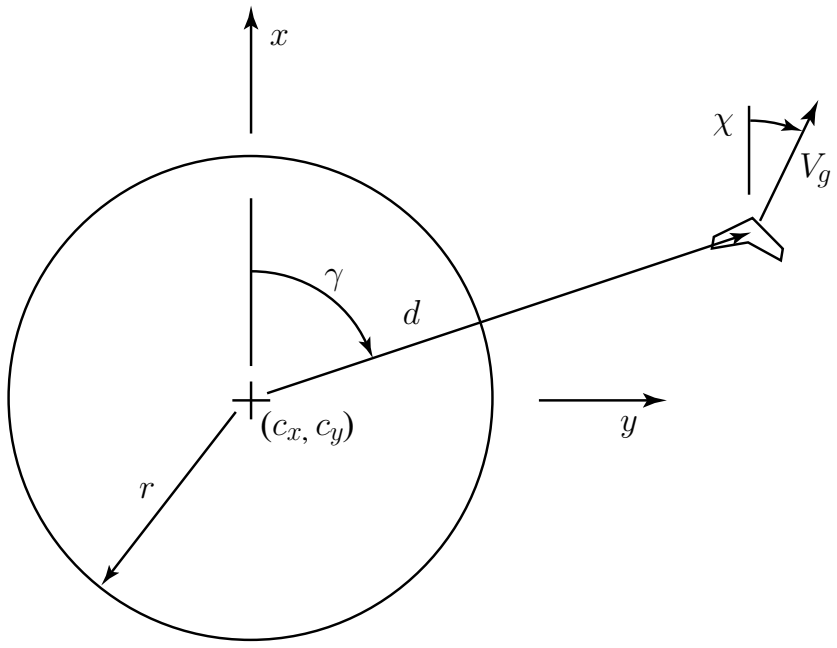

Fig. 6. Vector field geometry for orbit tracking. When the radial distance to the MAV is much greater than the orbit radius the desired course is calculated so that the MAV is directed toward the orbit center. As the radial distance becomes smaller, the desired course becomes tangential to the orbit.

course, respectively. We will again assume that the course dynamics are given by

$$
\dot{\chi}=\alpha\left(\chi^{c}-\chi\right) \text {. }
$$

Defining $\tilde{d}=d-r$ then we can argue that $\tilde{d} \rightarrow 0$ asymptotically when $\chi=\chi^{d}(d)$ by using the Lyapunov function $W_{1}=\frac{1}{2} \tilde{d}^{2}$, whose Lie derivative is

$$
\begin{aligned}
\dot{W}_{1} & =V_{g} \tilde{d} \cos \left(-\frac{\pi}{2}-\tan ^{-1}(k \tilde{d})\right) \\
& =-V_{g} \tilde{d} \sin \left(\tan ^{-1}(k \tilde{d})\right) .
\end{aligned}
$$

$\dot{W}_{1}$ is less than zero for $\tilde{d} \neq 0$ since the argument of $\sin$ is in the set $(-\pi / 2, \pi / 2)$ for all $\tilde{d}$, implying that $\tilde{d} \rightarrow 0$ asymptotically.

We will again use a sliding mode approach to render the set

$$
S=\left\{(\tilde{d}, \chi): \chi=\chi^{d}(d)\right\}
$$

positively invariant and to ensure that the system trajectory reaches $S$ in finite time. As before, define $\tilde{\chi}=\chi-\chi^{d}(d)$ and differentiate to obtain

$$
\begin{aligned}
\dot{\tilde{\chi}} & =\alpha\left(\chi^{c}-\chi\right)-\dot{\chi}^{d}(d) \\
& =\alpha\left(\chi^{c}-\chi\right)-\frac{V_{g}}{d} \sin (\chi-\gamma)+\beta V_{g} \cos (\chi-\gamma),
\end{aligned}
$$

where

$$
\beta=\frac{k}{1+(k \tilde{d})^{2}}
$$

has been defined for brevity. Letting $W_{2}=\frac{1}{2} \tilde{\chi}^{2}$ gives

$$
\dot{W}_{2}=\tilde{\chi}\left(\alpha\left(\chi^{c}-\chi\right)-\frac{V_{g}}{d} \sin (\chi-\gamma)+\beta V_{g} \cos (\chi-\gamma)\right) .
$$

If we choose the control signal as

$$
\chi^{c}=\chi+\frac{V_{g}}{\alpha d} \sin (\chi-\gamma)-\frac{\beta}{\alpha} V_{g} \cos (\chi-\gamma)-\frac{\kappa}{\alpha} \operatorname{sign}(\tilde{\chi}),
$$

where $\kappa>0$, then

$$
\dot{W}_{2} \leq-\kappa|\tilde{\chi}|
$$

from which we conclude that $\tilde{\chi} \rightarrow 0$ in finite time [12].

To avoid chattering in the orbit case we replace (19) with

$$
\chi^{c}=\chi+\frac{V_{g}}{\alpha d} \sin (\chi-\gamma)-\frac{\beta}{\alpha} V_{g} \cos (\chi-\gamma)-\frac{\kappa}{\alpha} \operatorname{sat}\left(\frac{\tilde{\chi}}{\epsilon}\right),
$$

where $\epsilon>0$ defines the width of the boundary region around the sliding mode.

Theorem III.2 The system of equations given by (16) and (18), where $\chi^{c}$ is given by (20) and $\chi^{d}(d)$ is given by (15) is globally exponentially stable if

$$
\left(\frac{k}{\epsilon \mu}\right) \min \left\{\left(\frac{\rho \kappa}{V_{g}}\right),\left(\frac{\bar{d}}{2}\right)\right\}>1,
$$

where

$$
\mu \geq 2\left[1+\left(k \bar{d}^{2}\right)\right]^{\frac{3}{2}}
$$

and $\bar{d}$ is arbitrary.

Proof:

The proof follows a similar line of reasoning as the proof of Theorem III.1. If $|\tilde{\chi}| \geq \epsilon$, then the set $S_{\epsilon}=\{|\tilde{\chi}| \leq \epsilon\}$ is positively invariant and $\tilde{\chi}$ converges to $S_{\epsilon}$ in finite time. Inside the boundary region we use the Lyapunov function candidate

$$
W=\frac{1}{2} \tilde{d}^{2}+\frac{1}{2} \rho \tilde{\chi}^{2}
$$

with $\rho>0$ to obtain

$$
\dot{W}=-\frac{\rho \kappa}{\epsilon} \tilde{\chi}^{2}+V_{g} \tilde{d} \sin \left(\hat{\chi}^{d}(d)+\tilde{\chi}\right)
$$

where $\hat{\chi}^{d}(d) \triangleq-\tan ^{-1}(k \tilde{d})$. Following steps that are similar to the proof of Theorem III.1 we get

$$
\begin{aligned}
\dot{W} & \leq-\frac{\rho \kappa}{\epsilon} \tilde{\chi}^{2}+2 V_{g}|\tilde{d}||\tilde{\chi}|+V_{g} \tilde{d} \sin \left(\hat{\chi}^{d}(d)\right) \\
& =-\frac{\rho \kappa}{\epsilon} \tilde{\chi}^{2}+2 V_{g}|\tilde{d}||\tilde{\chi}|-V_{g} \tilde{d} \sin \left(\tan ^{-1}(k \tilde{d})\right) .
\end{aligned}
$$

Letting

$$
\phi(\tilde{d})=\tilde{d} \sin \left(\tan ^{-1}(k \tilde{d})\right),
$$

and

$$
\varphi(\tilde{d})= \begin{cases}\frac{k}{\mu} \tilde{d}^{2} & \text { if }|\tilde{d}| \leq \bar{d} \\ \frac{k \bar{d}}{\mu}(2|\tilde{d}|-\bar{d}) & \text { if }|\tilde{d}|>\bar{d}\end{cases}
$$


where $\bar{d}$ is arbitrary, we can show that $0<\varphi(\tilde{d}) \leq \phi(\tilde{d})$ if $\mu \geq 2\left[1+\left(k \bar{d}^{2}\right)\right]^{\frac{3}{2}}$. From this we find that for $|\tilde{d}| \leq \bar{d}$,

$$
\begin{aligned}
\dot{W} & \leq-\frac{\rho \kappa}{\epsilon} \tilde{\chi}^{2}+2 V_{g}|\tilde{d}||\tilde{\chi}|-\frac{V_{g} k}{\mu} \tilde{d}^{2} \\
& =-V_{g}\left(\begin{array}{ll}
|\tilde{\chi}| & |\tilde{d}|)
\end{array}\right)\left(\begin{array}{cc}
\frac{\rho \kappa}{\epsilon V_{g}} & -1 \\
-1 & \frac{k}{\mu}
\end{array}\right)\left(\begin{array}{c}
|\tilde{\chi}| \\
|\tilde{d}|
\end{array}\right)
\end{aligned}
$$

which is negative definite if

$$
\frac{\rho \kappa k}{V_{g} \epsilon \mu}>1
$$

For $|\tilde{d}|>\bar{d}$, we get

$$
\dot{W} \leq-\frac{\rho \kappa}{\epsilon} \tilde{\chi}^{2}+2 V_{g}|\tilde{d}||\tilde{\chi}|-\frac{V_{g} k \bar{d}}{\mu}(2|\tilde{d}|-\bar{d})
$$

which is $<0$ if

$$
\frac{k \bar{d}}{2 \epsilon \mu}>1
$$

The combination of (22) and (23) leads to (21).

\section{Combining Straight Lines and Orbits}

Many paths planned for MAV flight can be approximated by combinations of straight-line segments and circular arcs [13]. Figure 7 shows how combined paths can be utilized with waypoint planning to fly paths that preserve equal path lengths, fly directly over the waypoints, or turn in order to minimize flight time. There are also a number of other situations where a combination would be desirable. For example, following a perimeter with irregular geometry could be done effectively by approximating its geometry with a series of lines and arcs.

When combining straight and arc segments, an approach for constructing the vector field must be developed. In order to avoid the possibility of multiple sinks, dead zones, and singularities that are inherent in the combination of vector fields, only the vector field for the current segment or arc to be followed is calculated. For a multi-segmented path, the vector field changes each time the MAV reaches the end of a segment or arc. Once the MAV has reached the end of a segment or arc, the entire vector field changes to direct the MAV onto the next segment or arc. No two fields are combined, thus eliminating any issues related to the combining of fields.

The method for determining when to change the vector field must be specified. There are a number of methods for doing this. One way is to detect when the MAV is within a predetermined distance from the end of the segment or arc. This works well for transitioning out of a straight path segment. For transitioning out of an arc, monitoring the angular travel of the MAV has proven successful. Using this approach, the MAV transitions to the next path segment when the angle through which the MAV has flown is equal to the included angle of the arc.

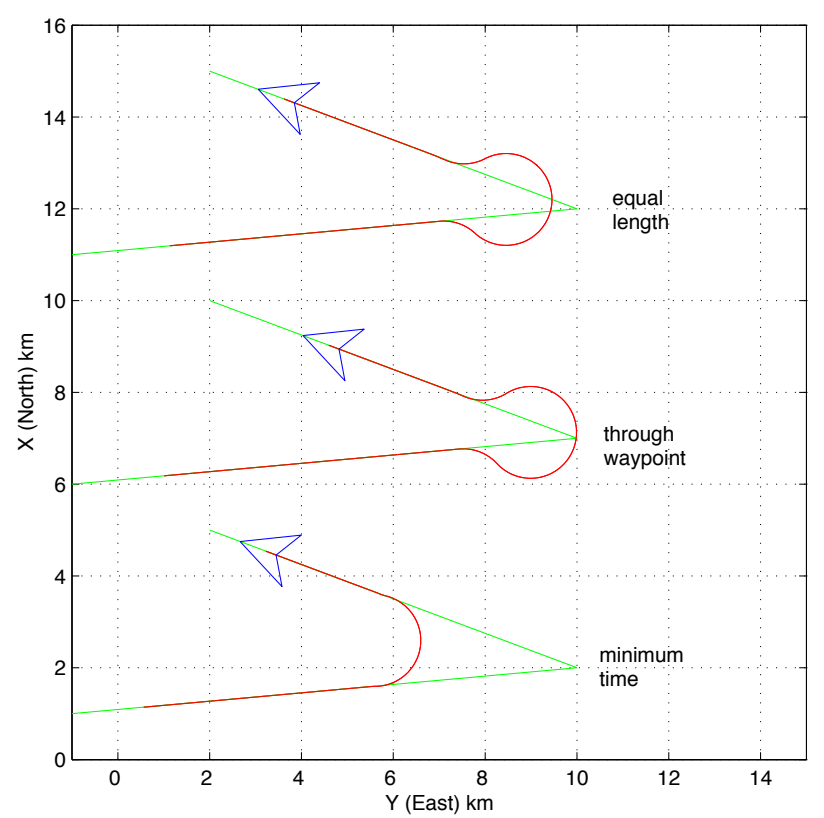

Fig. 7. Straight line paths and circular orbits can be combined to produce a variety of paths. The top figure shows the combination of two straight line paths and three circular orbits that are arranged so that if the MAV is on the path, the path length is equal to the original waypoint path. The middle figure arranges the orbits so that the desired path transitions over the waypoint. In the bottom figure a single orbit is used to transition between straight line segments.

\section{RESULTS AND DisCUSSION}

\section{A. Hardware Testbed}

BYU has developed a reliable and robust platform for testing unmanned air vehicles [14], [15], [16]. Figures 8 through 10 show the key elements of the testbed. Figure 8 shows BYU's Kestrel autopilot which is equipped with a Rabbit $340029 \mathrm{MHz}$ processor, rate gyros, accelerometers, absolute and differential pressure sensors. The autopilot measures $3.8 \times 5.1 \times 1.9 \mathrm{~cm}$ and weighs 17 grams.

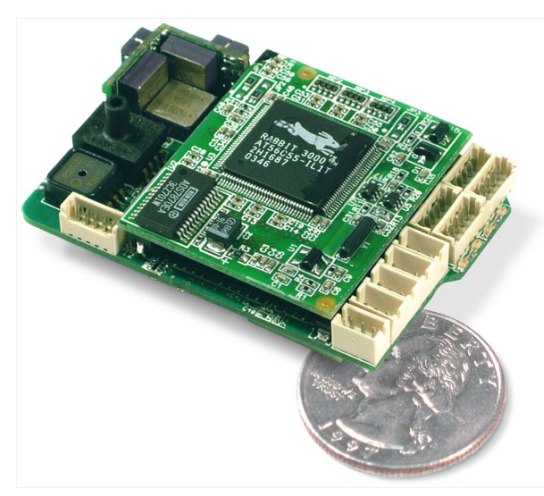

Fig. 8. The Kestrel autopilot system developed at BYU.

Figure 9 shows the airframe used for the flight tests reported in this paper. The airframe is a 1.2 meter wingspan Zagi XS EPP foam flying wing, which was selected for its durability, ease of component installation, and flight characteristics. Embedded in the airframe are the Kestrel autopilot, batteries, a 


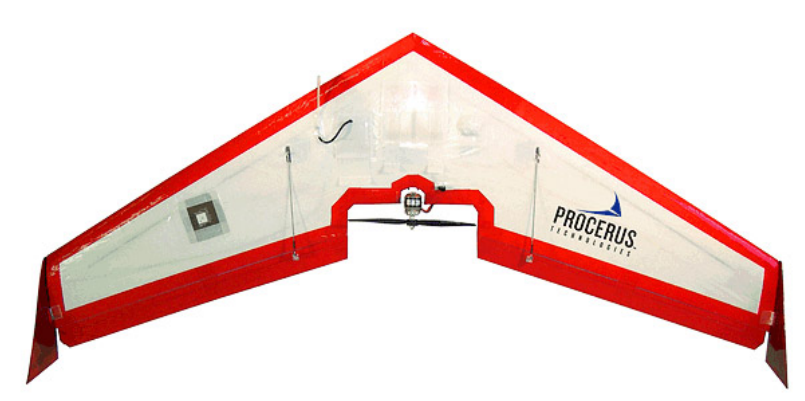

Fig. 9. Zagi foam flying wing airframe.

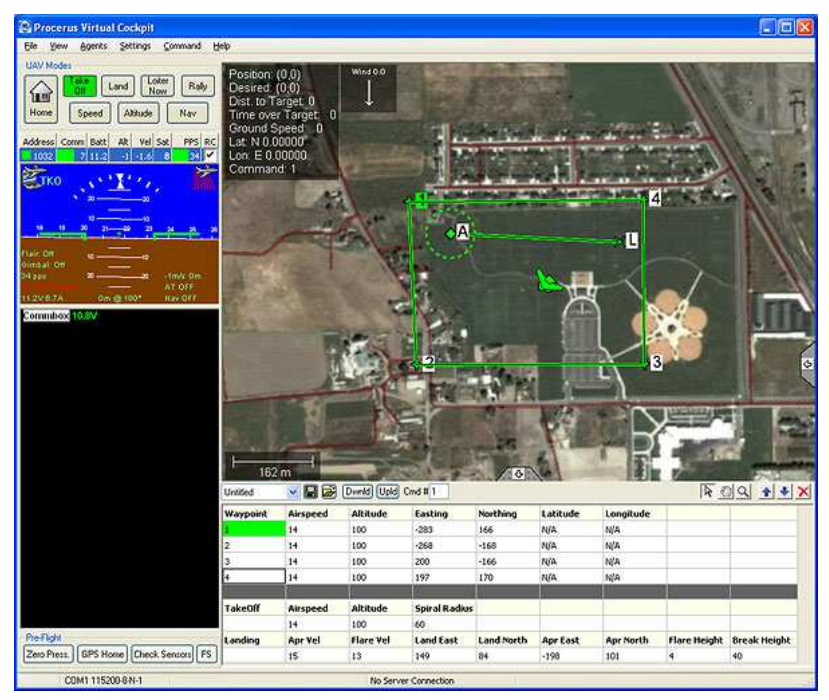

Fig. 10. Screen shot of the Virtual Cockpit software used to interact with the MAV.

$1000 \mathrm{~mW}, 900 \mathrm{MHz}$ radio modem, a GPS receiver, a video transmitter, and a small analog camera.

Figure 10 shows a screen shot of the Virtual Cockpit software that interfaces through a communication box to the MAVs. Virtual Cockpit allows the user to control the behavior of the MAV by changing control gains, specifying waypoints, or changing experimental objectives. Telemetry from the MAV is displayed in the Virtual Cockpit in real time allowing the user to monitor the progress of the experiment.

To implement the straight-line and orbit tracking control laws, several control parameters must be specified. Table I specifies the parameters used for the experimental results presented. With these values, the stability conditions specified by (13) and (21) are satisfied provided that $\rho$ is selected to scale the following and course errors so that they are of similar magnitudes during path following. For example, in the straight-line case, choosing $\rho$ so that

$$
\rho \approx \frac{y_{\max }^{2}}{\tilde{\chi}_{\max }^{2}}=\frac{y_{\max }^{2}}{\pi^{2}}
$$

ensures that the stability condition is a meaningful indicator of system behavior.
TABLE I

Control Parameter Values

\begin{tabular}{|c|c|c|}
\hline parameter & value & equation \\
\hline \hline$\alpha$ & $1.65 \mathrm{rad} / \mathrm{s}$ & $(7),(18)$ \\
$k$ & $0.02 \mathrm{~m}^{-1}$ & $(8),(15)$ \\
$\chi^{\infty}$ & $\pi / 2 \mathrm{rad}^{2}$ & $(8)$ \\
$\kappa$ & $\pi / 2 \mathrm{rad}^{2} / \mathrm{s}$ & $(12),(20)$ \\
$\epsilon$ & $1.0 \mathrm{rad}$ & $(12),(20)$ \\
\hline
\end{tabular}

\section{B. Experimental Results}

To demonstrate the path following abilities enabled by the vector field algorithm, MAVs were commanded to fly a variety of paths composed of straight lines, orbits, and combinations of straight lines and circular arcs. Experiments were conducted on three separate days. The majority of the results (which are presented first) were collected on a relatively calm day with the average wind speed measuring $0.9 \mathrm{~m} / \mathrm{s}$ from 230 degrees southwest. The commanded airspeed was $13 \mathrm{~m} / \mathrm{s}$, thus for these tests the average wind speed was 7 percent of the commanded airspeed. Results were also gathered on two highwind days: one with an average wind speed of $9 \mathrm{~m} / \mathrm{s}$ and another with an average wind speed of $6 \mathrm{~m} / \mathrm{s}$.

The position of the MAV was measured using the on-board GPS unit. The bias error associated with the GPS measurement is approximately $10 \mathrm{~m}$ and is virtually unchanging over the duration of the flight experiments. Random errors are in the range of 2 to $3 \mathrm{~m}$. The path-following errors reported are the calculated by computing the lateral distance between desired path and the location of the MAV as measured by GPS.

To illustrate orbit following with the vector field algorithm, the MAV was commanded to fly a series of concentric orbits with varying radii. The results are shown in Figure 11. The mean lateral path error for the $150 \mathrm{~m}$ orbit was $0.58 \mathrm{~m}$, while the standard deviation of the path error was $0.42 \mathrm{~m}$. For the $100 \mathrm{~m}$ orbit the mean path error was $0.65 \mathrm{~m}$ and the standard deviation of the path error was $0.46 \mathrm{~m}$. For the $70 \mathrm{~m}$ orbit, the mean and standard deviation of the lateral error was $1.9 \mathrm{~m}$ and $1.1 \mathrm{~m}$ respectively. For the larger orbits, the mean errors are about half of the wingspan of the MAV. The data indicate that tight tracking is more difficult to achieve as the orbit radius decreases. This is expected since control adjustments for loss in altitude in turns must be mixed with those used to control the lateral error and since the states associated with a smaller orbit are further away from the nominal wings-level trim condition.

Figure 12 illustrates the ability of the MAV to follow straight line segments with acute angles. Excluding the transient errors from the turns, the mean following error on the straight-line portions of the path was $0.74 \mathrm{~m}$ with a standard deviation of $0.66 \mathrm{~m}$.

A combination of the straight-line and arc-following methods was also tested. The techniques described in Section III-C were implemented and the results are plotted in Figure 13. The thicker line represents the desired path that was planned to equalize the straight-line and smoothed path lengths. The mean path following error and standard deviation were $3.6 \mathrm{~m}$ and $5.1 \mathrm{~m}$ respectively. Although the transitions from the straight 


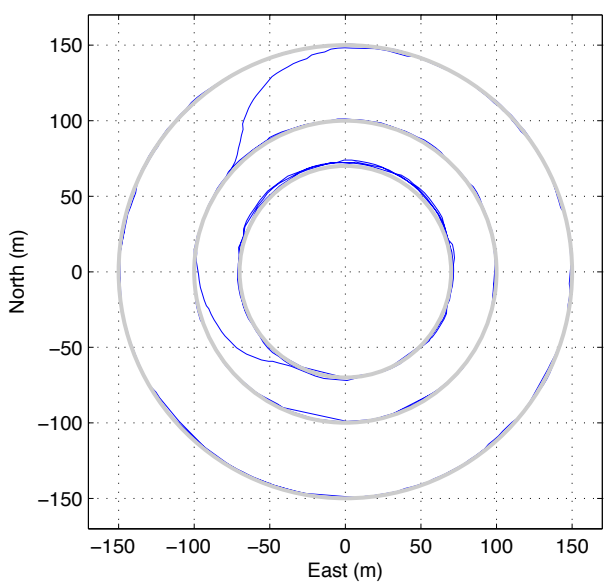

Fig. 11. Telemetry plot for orbits with radii of 150,100 , and $70 \mathrm{~m}$.

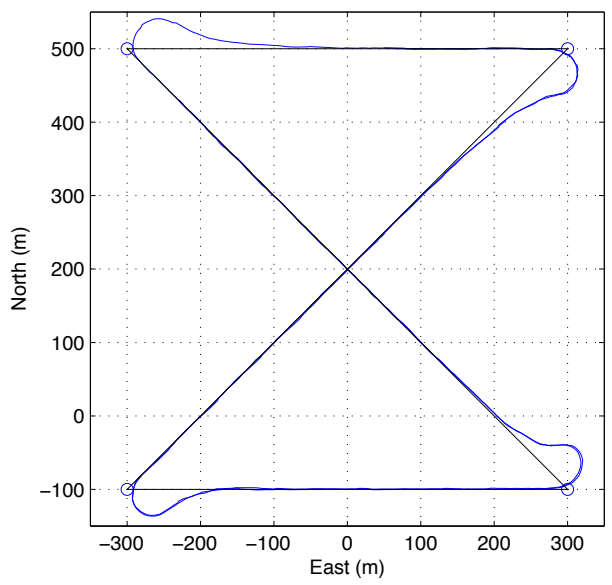

Fig. 12. Telemetry plot for straight line following.

line to the orbit portions show some lateral following errors, the length of the path flown and the desired length are similar. The length of the straight-line path shown in Figure 13 was $2897 \mathrm{~m}$. The actual distance flown was $27 \mathrm{~m}$ less than the desired distance, which is an error of only 0.93 percent.

To further test the robustness and capabilities of the proposed path-following algorithms, many other types of paths have been flown. The path shown in Figure 14 illustrates both obtuse and acute angles and the decision of the path follower to cut the corners of the obtuse angles and flare out and around on the acute angles. Considering the path following error over the full path gave a mean error of $3.6 \mathrm{~m}$ and a standard deviation of $4.7 \mathrm{~m}$. Figure 15 shows a path representative of an urban scenario. Although these are actual flight results, the terrain is synthetic. The straight-line follower was used to follow this path. The mean lateral error over the full path was $3.4 \mathrm{~m}$, while the standard deviation was $4.8 \mathrm{~m}$.

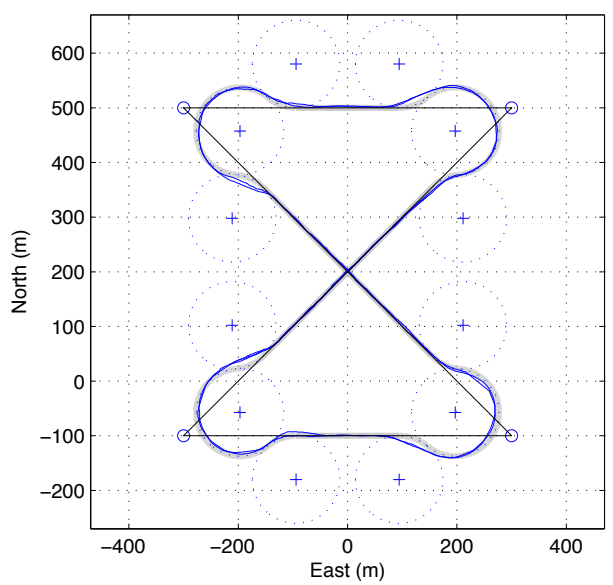

Fig. 13. Telemetry plot for equal path length following.

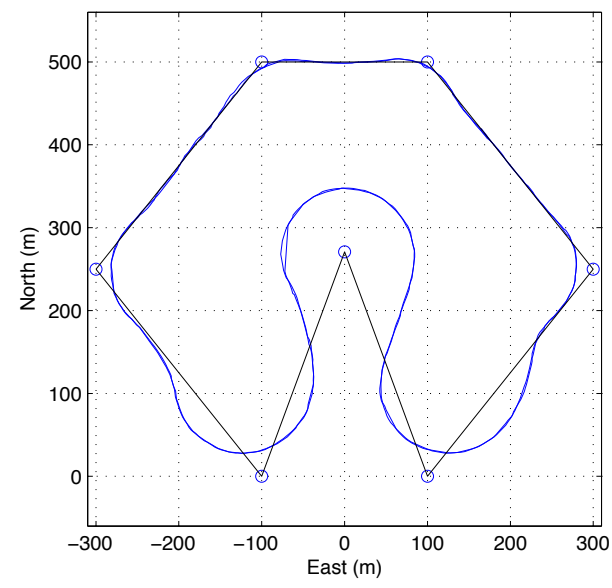

Fig. 14. Combination of equal path length and corner cutting following.

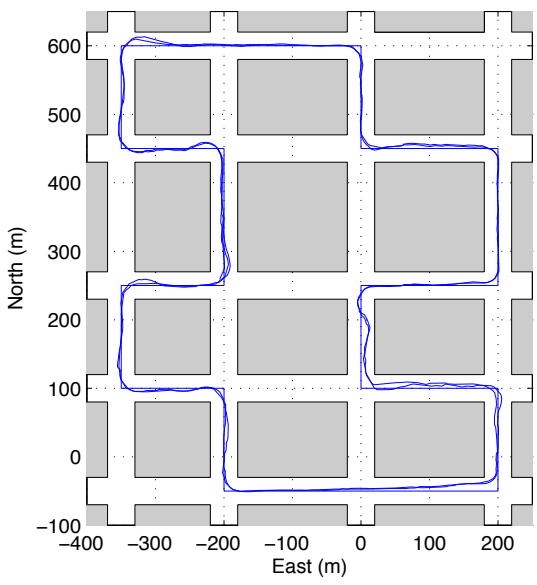

Fig. 15. Urban terrain following using straight line following.

To demonstrate the feasibility of the approach for high wind conditions, an orbit path and a straight-line path were flown on days where the average wind speeds were $9 \mathrm{~m} / \mathrm{s}$ and $6 \mathrm{~m} / \mathrm{s}$ respectively. A plot of the orbit results is shown in Figure 16. 
For these results, the high wind required an airspeed of $18 \mathrm{~m} / \mathrm{s}$ to enable the MAV to make steady progress upwind. The wind speed was approximately 50 percent of the commanded airspeed. Under these conditions, the mean following error was $0.5 \mathrm{~m}$ with a standard deviation of $4.2 \mathrm{~m}$.

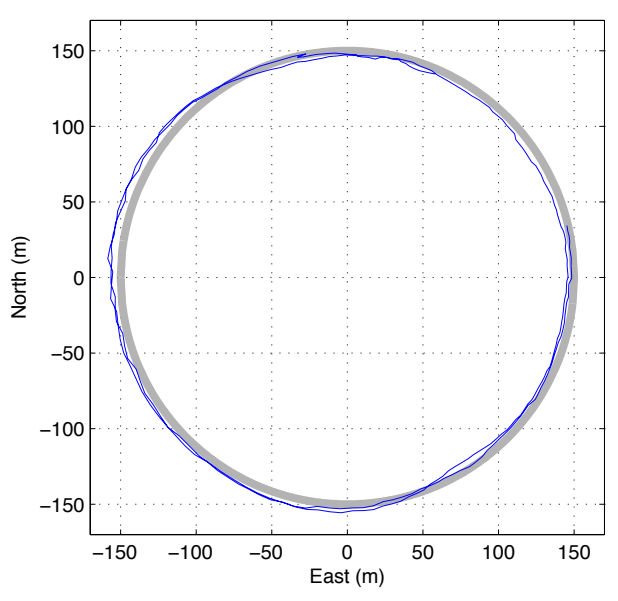

Fig. 16. Orbit following in high-wind conditions. Wind speed $50 \%$ of commanded airspeed.

Straight-line path following results gathered under highwind conditions are shown in Figure 17. The wind was measured at $6 \mathrm{~m} / \mathrm{s}$ coming from the south. The commanded airspeed was $13 \mathrm{~m} / \mathrm{s}$. As expected, the largest errors occur during the down-wind portions of a turn. In these situations, the groundspeed of the MAV is high and the minimum turn radius, which is governed by roll-angle limits, becomes larger. It can be seen that on the straight line portions of the path, the following errors are small. In this case, the mean following error was $0.5 \mathrm{~m}$, while the standard deviation of the error was $1.2 \mathrm{~m}$ along the straight portions of the path.

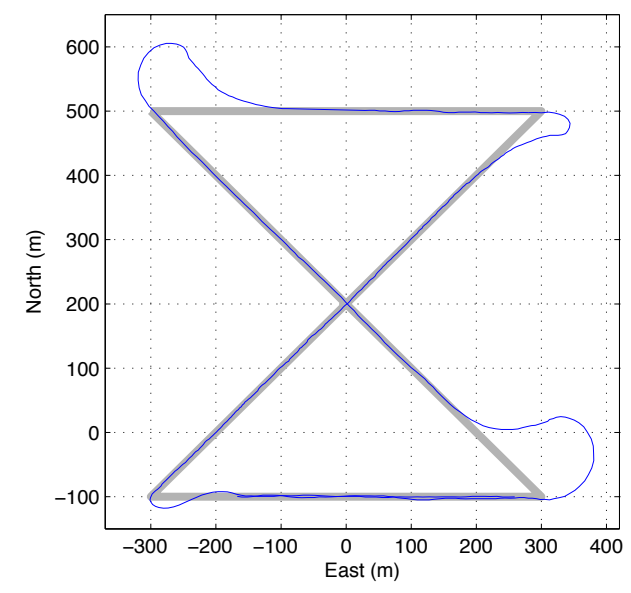

Fig. 17. Straight-line following in high-wind conditions. Wind speed $46 \%$ of commanded airspeed.

\section{CONCLUSIONS}

In this paper, a new method for MAV path following, based on the concept of vector fields, has been introduced. Using Lyapunov stability criteria, it has been shown that the vector field approach provides asymptotic following for straight-line and circular paths in the presence of constant wind disturbances.

The effectiveness of the vector field method has been demonstrated experimentally using a fixed-wing MAV. For both straight-line and circular paths, following errors averaged less than one wingspan in steady straight-line or orbit paths and less than three wingspans for paths involving frequent transitions between straight-line and arc segments.

\section{ACKNOWLEDGMENTS}

The work was supported by AFOSR grant number FA955004-1-0209.

\section{REFERENCES}

[1] S. Park, J. Deyst, and J. How, "A new nonlinear guidance logic for trajectory tracking," in Proceedings of the AIAA Guidance, Navigation and Control Conference, August 2004, AIAA-2004-4900.

[2] I. Kaminer, A. Pascoal, E. Hallberg, and C. Silvestre, "Trajectory tracking for autonomous vehicles: An integrated approach to guidance and control," AIAA Journal of Guidance, Control and Dynamics, vol. 21, no. 1, pp. 29-38, 1998.

[3] P. Aguiar, D. Dačić, J. Hespanha, and P. Kokotivić, "Path-following or reference-tracking? An answer relaxing the limits to performance," in Proceedings of IAV2004, 5th IFAC/EURON Symposium on Intelligent Autonomous Vehicles, Lisbon, Portugal, 2004.

[4] P. Aguiar, J. Hespanha, and P. Kokotović, "Path-following for nonminimum phase systems removes performance limitations," IEEE Transactions on Automatic Control, vol. 50, no. 2, pp. 234-239, 2005.

[5] J. Hauser and R. Hindman, "Maneuver regulation from trajectory tracking: Feedback linearizable systems," in Proceedings of the IFAC Symposium on Nonlinear Control Systems Design, Tahoe City, CA, June 1995, pp. 595-600.

[6] P. Encarnação and A. Pascoal, "Combined trajectory tracking and path following: An application to the coordinated control of marine craft," in Proceedings of the IEEE Conference on Decision and Control, Orlando, FL, 2001, pp. 964-969.

[7] R. Skjetne, T. Fossen, and P. Kokotović, "Robust output maneuvering for a class of nonlinear systems," Automatica, vol. 40, pp. 373-383, 2004.

[8] R. Rysdyk, "UAV path following for constant line-of-sight," in Proceedings of the 2nd AIAA Unmanned Unlimited Conference, 2003, aIAA2003-6626.

[9] O. Khatib, "Real-time obstacle avoidance for manipulators and mobile robots," in Proceedings of the IEEE International Conference on Robotics and Automation, vol. 2, 1985, pp. 500-505.

[10] K. Sigurd and J. P. How, "UAV trajectory design using total field collision avoidance," in Proceedings of the AIAA Guidance, Navigation and Control Conference, August 2003.

[11] E. Frew and D. Lawrence, "Cooperative stand-off tracking of moving targets by a team of autonomous aircraft," in Proceedings of the AIAA Guidance, Navigation and Control Conference, August 2005, AIAA2005-6363.

[12] H. K. Khalil, Nonlinear Systems, 3rd ed. Upper Saddle River, NJ: Prentice Hall, 2002.

[13] E. Anderson, R. Beard, and T. McLain, "Real-time dynamic trajectory smoothing for unmanned air vehicles," IEEE Transactions on Control Systems Technology, vol. 13, no. 3, pp. 471-477, 2005.

[14] “The Kestrel autopilot. Procerus Technologies." http://procerusuav.com/.

[15] R. Beard, D. Kingston, M. Quigley, D. Snyder, R. Christiansen, W. Johnson, T. McLain, and M. Goodrich, "Autonomous vehicle technologies for small fixed wing UAVs," AIAA Journal of Aerospace, Computing, Information, and Communication, vol. 2, no. 1, pp. 92-108, January 2005. 
[16] R. W. Beard, D. Lee, S. Thakoor, and S. Zornetzer, "A new approach to observation of descent and landing of future Mars mission using bioinspired technology innovations," AIAA Journal of Aerospace Computing, Information, and Communication, vol. 2, no. 1, pp. 65-91, January 2005. 